\title{
Front form and point form formulation of predictive relativistic mechanics. Noninteraction theorems
}

\author{
$X$. Jaen and A. Molina \\ Grup de Relativitat de la Secció de Física SCC (IEC), Departament de Física Teòrica, Universitat de \\ Barcelona, Barcelona, Spain \\ V. Iranzo \\ Grup de Relativitat de la Secció de Física SCC (IEC), Universitat Politècnica de Catalunya (ETSECCP), \\ Barcelona, Spain
}

(Received 14 September 1984; accepted for publication 20 September 1985)

The front form and the point form of dynamics are studied in the framework of predictive relativistic mechanics. The noninteraction theorem is proved when a Poincaré-invariant Hamiltonian formulation with canonical position coordinates is required.

\section{INTRODUCTION}

Instantaneous relativistic dynamics of particles with direct interaction was initiated in a celebrated paper by Dirac, ${ }^{1}$ entitled "Forms of relativistic dynamics." The line of thought set up there was further developed by Bakanjian, Thomas, and Foldy, ${ }^{2}$ in the framework that Dirac had called "instant form."

The subsequent development of the theory met the important drawback of the so-called "no-interaction theorem." "In general terms, it states that, if the position coordinates of the particles are to be canonical coordinates, and the particle worldlines must be Poincaré invariant, then the only systems that are compatible with both requirements are those consisting of free particles.

One attempt to circumvent this problem was initiated by Currie, ${ }^{4}$ and later on it has generated a rather wide stream of literature, which is known as predictive relativistic mechanics (and maybe, it should be called an instant form of PRM). It consists, first, in giving up the Hamiltonian formalism, which was taken for granted in former approaches, and starting from a more elementary level. The fundamental assumptions in predictive relativistic mechanics are (i) the equations of motion of the particles are Newton-like, that is, the acceleration of each particle is a given function of positions and velocities of all particles; and (ii) Poincaré invariance, which is understood to mean two things: the acceleration functions must be formally the same in all inertial reference frames, and particle worldlines must be Poincaré invariant. These requirements imply that some condition (the so-called Currie-Hill equations ${ }^{5}$ ) must be fulfilled by the acceleration functions. In addition, they also ensure the possibility of setting up a realization of the Poincare algebra ${ }^{6}$ on the system's tangent space (the one spanned by positions and velocities). Now, the no-interaction result can be obtained again ${ }^{7}$ if one seeks for a Hamiltonian formalism such that the aforementioned realization of the Poincaré algebra is canonical, and the position coordinates can be taken as canonical.

As far as we know, all proofs of the no-interaction theorem hitherto derived share a common feature, namely, physical variables are assumed to be simultaneous in a given inertial frame. This is a specific trait of the "instant form" of relativistic dynamics. However, in the pioneering paper by
Dirac, ${ }^{1}$ two other possibilities were considered, namely, the "front form" and the "point form" (in fact, a later paper by Leutwyler and Stern ${ }^{7}$ increases that number by two more "forms").

One then wonders whether the no-interaction theorem, or a related result, also holds in these two alternative forms of relativistic dynamics. Although this is, indeed, an interesting point to be elucidated, it seems not to have been proven yet. Indeed, in a relatively recent paper by Leutwyler and Stern ${ }^{7}$ we can find the following sentence: "Although this no go theorem has been established only for theories of class (i) (i.e., the "instant form" of relativistic dynamics) it likely also holds for the remaining four forms of Hamiltonian dynamics."

In the present paper we intend to give an answer to the question that is more or less implicit in the quoted sentence, and derive a no-interaction theorem in the front form as well as in the point form. The master lines of our proof are the same as those of the proof given by Hill ${ }^{5}$ for the no-interaction theorem in the "instant form."

In a natural way, the paper is divided in two parts. The first one (Secs. II and III) is devoted to the front form, and the second one (Secs. IV and V) to the point form. Besides, each part is organized in two sections: one devoted to develop what could be called the front (resp. point) form of predictive relativistic mechanics, and the other to prove the nointeraction theorem.

\section{FRONT FORM OF PREDICTIVE RELATIVISTIC MECHANICS}

In the instant form of predictive relativistic mechanics ${ }^{8}$ (which has been its only formulation up to now), the extended configuration space of $N$ spinless particles is spanned by the $3 N+1$ variables: $t, x_{b}^{i}, b=1, \ldots, N, i=1,2,3$; where the evolution parameter is the time coordinate as measured in a given inertial frame, and the $x_{b}^{i}$ are the space coordinates of the event determined by the intersection of the worldline of particle $b$ and the space hyperplane $x^{4}=t$.

The equations of motion are then required to be secondorder differential equations, that is,

$$
\frac{d^{2} x_{b}^{i}}{d t^{2}}=a_{b}^{i}\left(x_{c}^{i}, v_{e}^{k}, t\right), \quad \frac{d x_{b}^{i}}{d t}=v_{b}^{i} .
$$


Thus the space of initial data is spanned by the following $6 N+1$ variables:

$$
t, x_{b}^{i}, v_{c}^{k}, \quad b, c=1, \ldots, N, \quad i, k=1,2,3 .
$$

If the space hyperplanes $x^{4}=t$ characterize the instant form, likewise the null hyperplanes $x^{3}+x^{4}=\lambda$ will play a central role in the front form of relativistic dynamics (here $t$ and $\lambda$ are two real parameters). So, the extended configuration space in the front form will be coordinated by the $3 N+1$ variables: $\lambda, x_{b}^{i}, b=1, \ldots, N, i=1,2,3$; where $\lambda$ is the evolution parameter and $x_{b}^{i}, i=1,2,3$, are the space coordinates of the event where the worldline of particle $b$ meets the null hyperplane

$$
x^{3}+x^{4}=\lambda,
$$

or, using the notation introduced in (A5),

$$
x^{+}=\lambda
$$

(the same value of $\lambda$ for all particles).

For convenience, our configuration space coordinates will be (see Appendix A)

$$
x_{a}^{A}, \quad a=1, \ldots, N, \quad A=1,2,-,
$$

rather than the Cartesian $x_{a}^{i}, i=1,2,3$.

We now require the motion to be governed by a secondorder differential system,

$$
\begin{aligned}
\frac{d^{2} x_{b}^{A}}{d \lambda^{2}} & =a_{b}^{A}\left(x_{a}^{B}, v_{c}^{D} ; \lambda\right), \quad \frac{d x^{A}}{d \lambda}=v_{b}^{A}, \\
a, b, c & =1, \ldots, N, \quad A, B, D=1,2,-.
\end{aligned}
$$

For every given solution of (2.3), we have a set of $N$ worldlines describing the history of the system. Indeed, if $\varphi_{b}^{A}\left(x_{a}^{B}, v_{c}^{D} ; \lambda\right), A, B, D=1,2,-, a, b, c=1, \ldots, N$, is the solution of (2.3) corresponding to the initial data

$$
\begin{aligned}
& \varphi_{b}^{A}\left(x_{a}^{B}, v_{c}^{D} ; 0\right)=x_{b}^{A}, \\
& \frac{\partial \varphi_{b}^{A}}{\partial \lambda}\left(x_{a}^{B}, v_{c}^{D} ; 0\right)=v_{b}^{A},
\end{aligned}
$$

then, according to (2.2) and (A5), the worldline $x_{b}^{\mu}(\lambda)$ of particle $b$ will be taken as

$$
\begin{aligned}
& x_{b}^{i}(\lambda)=\varphi_{b}^{i}\left(x_{a}^{B}, v_{c}^{D} ; \lambda\right), \quad i=1,2, \\
& x_{b}^{3}(\lambda)=\lambda / 2+\varphi_{b}^{-}\left(x_{a}^{B}, v_{c}^{D} ; \lambda\right), \\
& x_{b}^{4}(\lambda)=\lambda / 2-\varphi_{b}^{-}\left(x_{a}^{B}, v_{c}^{D} ; \lambda\right),
\end{aligned}
$$

which in the adapted coordinates (A4) reads

$$
x_{b}^{A}(\lambda)=\varphi_{b}^{A}\left(x_{a}^{B}, v_{c}^{D} ; \lambda\right), \quad x_{b}^{+}(\lambda)=\lambda .
$$

Similarly to the instant form description, the principle of relativity will be used at two different levels. First, the "acceleration" functions $a_{b}^{A}$ on the right of Eq. (2.3) must have the same form in every inertial frame. And second, the dynamic system must be worldline invariant. The latter requirement means the same as in the instant form case, namely, that if $S$ and $S^{\prime}$ are two inertial frames related to each other by a Poincaré transformation $\left(\mathscr{L}_{\frac{\bar{A}}{B}}^{\bar{B}}, \mathscr{A}^{\bar{D}}\right), \bar{A}, \bar{B}, \bar{D}$ $=1,2,-,+$ - see the Appendix - and $x_{b}^{\bar{A}}(\lambda), b=1, \ldots, N$, $\vec{A}=1,2,-,+$ are the worldlines of the particles in the frame $S$, when the system starts from a given set of initial data $Z_{0} \equiv\left(x_{a}^{A}, v_{c}^{D}\right), a, c=1, \ldots, N, A, D=1,2,-$; then the
Poincaré-transformed worldlines

$$
x_{b}^{\prime \bar{A}}(\lambda)=\mathscr{L}_{\bar{B}}^{\bar{A}}\left(x_{b}^{\bar{B}}(\lambda)-A^{\bar{B}}\right)
$$

must be obtained in the frame $S^{\prime}$, when the system starts from the transformed set of initial data $z_{0}^{\prime} \equiv\left(x_{a}^{\prime A}, v_{c}^{\prime D}\right)$.

Thus, as happens in the instant form, the mapping $z_{0} \rightarrow z_{0}^{\prime}$ defines the induced action of Poincaré transformation $\left(\mathscr{L}_{\bar{B}}^{\bar{A}}, \mathscr{A}^{\bar{D}}\right)$ on the space of initial data.

In terms of the adapted coordinates, this mapping reads

$$
\begin{aligned}
& x_{a}^{\prime A}=f_{a}^{A}\left(x_{b}^{B}, v_{c}^{D} ; \epsilon^{\bar{E}}, \epsilon^{\overline{F G}}\right), \\
& v_{a}^{\prime A}=g_{a}^{A}\left(x_{b}^{B}, v_{c}^{D} ; \epsilon^{\bar{E}}, \epsilon^{\overline{F G}}\right),
\end{aligned}
$$

where $\epsilon^{\bar{E}}, \epsilon^{\overline{F G}}, \bar{E}, \bar{F}, \bar{G}=1,2,-,+$ denote the parameters characterizing the specific Poincare transformation-see Eq. (A11).

The infinitesimal generators are then obtained in the usual way:

$$
\mathbf{P}_{\bar{E}}=\frac{\partial f_{a}^{A}}{\partial \epsilon^{\bar{E}}}\left(x_{b}^{B}, v_{c}^{D} ; 0,0\right) \frac{\partial}{\partial x_{a}^{A}}+\frac{\partial g_{b}^{B}}{\partial \bar{\epsilon}^{\bar{E}}}\left(x_{b}^{B}, v_{c}^{D}, 0,0\right) \frac{\partial}{\partial v_{b}^{B}},
$$

$$
\mathbf{J}_{\overline{E F}}=\frac{\partial f_{a}^{A}}{\partial \epsilon^{\overline{E F}}}\left(x_{b}^{B}, v_{c}^{D} ; 0,0\right) \frac{\partial}{\partial x_{a}^{A}}+\frac{\partial g_{b}^{B}}{\partial \epsilon^{\overline{E F}}}\left(x_{b}^{B}, v_{c}^{D}, 0,0\right) \frac{\partial}{\partial v_{b}^{B}} .
$$

To obtain the specific expressions for these generators, we shall work out the condition of worldline invariance (2.7), together with the worldline equations (2.6). Introducing the latter into both sides of (2.7), we obtain

$$
\begin{aligned}
\varphi_{a}^{A}\left(z_{0}^{\prime}, \lambda_{a}\left(z_{0}, \lambda\right)\right)= & \operatorname{TX} \mathscr{L}_{E}^{A}\left[\varphi_{a}^{E}\left(z_{0}: \lambda\right)-\mathscr{A}^{E}\right] \\
& +\mathscr{L}_{+}^{A}\left[\lambda-\mathscr{A}^{+}\right], \quad A=1,2,-.
\end{aligned}
$$

It should be noticed that, since the "acceleration" functions have the same form in frame $S$ and in $S^{\prime}$, the same general solution $\varphi_{a}^{A}$ has been substituted into both sides of Eq. (2.7). However, whereas in the right-hand side we take the initial data $z_{0}=\left(x_{a}^{A}, v_{b}^{B}\right)$, which correspond to the frame $S$, in the left-hand side we have to put the transformed initial data $z_{0}^{\prime}=\left(x_{a}^{\prime A}, v_{b}^{\prime B}\right)$ which correspond to the worldlines as viewed from the frame $S^{\prime}$. Moreover, the value of the evolution parameter in the left-hand side of Eq. (2.10), which we have written as $\lambda_{a}\left(z_{0}, \lambda\right)$, will be presumed different from the parameter $\lambda$ in the right-hand side. This is due to the fact that the worldline invariance only ensures that each worldline transforms into another one as a whole, no matter how the respective parametrizations are related to each other.

In our case, the relation between parametrizations, i.e, the function $\lambda_{a}\left(z_{0}, \lambda\right)$, can be derived from the fact that the evolution parameter corresponds to the space-time coordinate $x^{+}$; so that we have

$\lambda_{a}\left(z_{0}, \lambda\right)=\mathscr{L}_{B}^{+}\left(\varphi_{a}^{B}\left(z_{0}, \lambda\right)-\mathscr{A}^{B}\right)+\mathscr{L}^{+}+\left(\lambda-\mathscr{A}^{+}\right)$.

By taking derivatives in (2.10) and (2.11) with respect to $\lambda$ we obtain the relation between velocities

$\dot{\varphi}_{a}^{A}\left(z_{0}^{\prime}, \lambda_{a}\left(z_{0}, \lambda\right)\right) \cdot \dot{\lambda}_{a}\left(z_{0}, \lambda\right)=\mathscr{L}_{E}^{A} \cdot \dot{\varphi}_{a}^{E}\left(z_{0}, \lambda\right)+\mathscr{L}_{+}^{A}$, 
with

$$
\dot{\lambda}_{a}\left(z_{0}, \lambda\right)=\mathscr{L}_{B}^{+} \cdot \dot{\varphi}_{a}^{B}\left(z_{0}, \lambda\right)+\mathscr{L}_{+}^{+},
$$

which, after one further differentiation, yields the relation between accelerations:

$$
\begin{gathered}
\ddot{\varphi}_{a}^{A}\left(z_{0}^{\prime}, \lambda_{a}\left(z_{0}, \lambda\right)\right) \dot{\lambda}_{a}^{2}\left(z_{a}, \lambda\right)+\dot{\varphi}_{a}^{A}\left(z_{0}^{\prime}, \lambda_{\mathrm{a}}\left(z_{0}, \lambda\right)\right) \ddot{\lambda}_{a}\left(z_{0}, \lambda\right) \\
=\mathscr{L}_{B}^{A} \ddot{\varphi}_{a}^{B}\left(z_{0}, \lambda\right)
\end{gathered}
$$

and

$$
\ddot{\lambda}_{a}\left(z_{0}, \lambda\right)=\mathscr{L}_{B}^{+} \ddot{\varphi}_{a}^{B}\left(z_{0}, \lambda\right) \text {. }
$$

Since $\varphi_{a}^{A}$ is a solution of the differential system (2.3), Eqs. (2.13) can be written as

$$
\begin{aligned}
& \mathscr{A}_{b}^{A}\left(\varphi_{b}^{B}\left(z_{0}^{\prime}, \lambda_{a}\left(z_{0}, \lambda\right)\right) ; \dot{\varphi}_{c}^{D}\left(z_{0}^{\prime}, \lambda_{a}\left(z_{0}, \lambda\right)\right) ;\right. \\
& \left.\quad \lambda_{a}\left(z_{0}, \lambda\right)\right) \cdot \dot{\lambda}_{a}^{2}\left(z_{0}, \lambda\right)+\dot{\varphi}_{a}^{A}\left(z_{0}^{\prime} ; \lambda_{a}\left(z_{0}, \lambda\right)\right) \cdot \ddot{\lambda}_{b}\left(z_{0}, \lambda\right) \\
& \quad=\mathscr{L}_{B}^{A} \mathscr{A}_{a}^{B}\left(\varphi_{b}^{D}\left(z_{0}, \lambda\right), \dot{\varphi}_{c}^{F}\left(z_{0}, \lambda\right), \lambda\right), \\
& \ddot{\lambda}_{a}\left(z_{0}, \lambda\right)=\mathscr{L}_{B}^{+} \mathscr{A}_{a}^{B}\left(\varphi_{b}^{D}\left(z_{0}, \lambda\right), \dot{\varphi}_{c}^{F}\left(z_{0}, \lambda\right), \lambda\right) .
\end{aligned}
$$

Equations (2.10)-(2.14), which hold for every value of $\lambda$ and for every Poincaré transformation $\left(\mathscr{L}_{\bar{B}}^{\bar{A}}, \mathscr{A}^{\bar{D}}\right)$, actually determine the functions $f_{a}^{A}, g_{b}^{B}$ in (2.8). Although, apart from a few trivial cases, it would be impossible to derive explicit expressions for such functions, the above equations permit us to obtain the infinitesimal generators in a rather straightforward way.

Indeed, introducing the infinitesimal expression (A12) for the Poincaré transformation $\left(\mathscr{L}_{\bar{B}}^{\bar{A}}, \mathscr{A}^{\bar{D}}\right)$ into Eqs. (2.10)-(2.12) and keeping first-order terms only, we obtain, after some manipulation,

$$
\begin{aligned}
& \mathbf{P}_{+}= \sum_{A=i, 2,-} \sum_{a=1}^{N}\left[v_{a}^{A} \frac{\partial}{\partial x_{a}^{A}}+a_{a}^{A} \frac{\partial}{\partial v_{a}^{A}}\right], \\
& \mathbf{P}_{A}=-\sum_{a=1}^{N} \frac{\partial}{\partial x_{a}^{A}}, A=1,2,-, \\
& \mathbf{J}_{+r}=-\sum_{a=1}^{N}\left\{\left(x_{a+} \delta_{r}^{A}+x_{a r} v_{a}^{A}\right) \frac{\partial}{\partial x_{a}^{A}}\right. \\
&\left.-\left(v_{a+} \delta_{r}^{A}+x_{a r} a_{a}^{A}+v_{a r} v_{a}^{A}\right) \frac{\partial}{\partial v_{a}^{A}}\right\}, \\
& \mathbf{J}_{+-}=-\sum_{a=1}^{N}\left\{x_{a+} \frac{\partial}{\partial x_{a}^{-}}+v_{a}+\frac{\partial}{\partial v_{a}^{-}}\right. \\
&\left.+\sum_{A=1,2,-} v_{a}^{A} \frac{\partial}{\partial v_{a}^{A}}\right\}, \\
& \mathbf{J}_{r-}= \sum_{a=1}^{N}\left\{-x_{a r} \frac{\partial}{\partial x_{a}^{-}}-v_{a r} \frac{\partial}{\partial v_{a}^{-}}+\frac{\partial}{\partial v_{a}^{r}}\right\}, \\
& \mathbf{J}_{1 z}=\sum_{a=1}^{N}\left\{-x_{a z} \frac{\partial}{\partial x_{a}^{1}}-x_{a 1} \frac{\partial}{\partial x_{a}^{2}}\right. \\
&\left.+v_{a z} \frac{\partial}{\partial v_{a}^{1}}-v_{a 1} \frac{\partial}{\partial v_{a}^{2}}\right\},
\end{aligned}
$$

where $r=1,2$.

Notice that, as expected, there are seven kinematical generators $\mathbf{P}_{A}, \mathbf{J}_{12}, \mathbf{J}_{r-}, \mathbf{J}_{+-}, A=1,2,-, r=1,2$; and three dynamical ones $\mathbf{P}_{+}, \mathbf{J}_{+}, r=1,2$; or Hamiltonians.

Now, by introducing the same infinitesimal Poincaré transformation (A12) into Eqs. (2.14), we obtain the set of differential equations

$$
\begin{aligned}
& \mathbf{P}_{A} a_{a}^{B}=0, \quad A, B=1,2,-, \\
& \mathbf{J}_{12} a_{a}^{B}=a_{a}^{2} \delta_{1}^{B}-a_{a}^{1} \delta_{2}^{B}, \\
& \mathbf{J}_{r-} a_{a}^{B}=-a_{a}^{r} \delta_{-}^{B}, \\
& \mathbf{J}_{+-} a_{a}^{B}=-\left(a_{a}^{-} \delta_{-}^{D}+z a_{a}^{D}\right), \\
& \mathbf{J}_{+} a_{a}^{B}=-z v_{a r} a_{a}^{B}-a_{a r} v_{a}^{B}-a_{a}+\delta_{r}^{B}-x_{a}^{r}\left(\mathbf{P}_{+} a_{a}^{B}\right),
\end{aligned}
$$

which play a similar role as the Currie-Hill conditions ${ }^{5}$ in the instant form of predictive relativistic mechanics.

\section{NO-INTERACTION THEOREM IN THE FRONT FORM OF DYNAMICS}

Let us now assume that we have a symplectic structure in the space of initial data, such that the "position variables" $x_{a}^{A}, A=1,2,-$, are canonical, i.e., the symplectic form is

$$
\sigma=d x_{a}^{A} \wedge d p_{A}^{a},
$$

where $p_{A}^{a}=p_{A}^{a}\left(x_{b}^{B}, v_{c}^{D}\right), a, b, c=1, \ldots, N, A, B, D=1,2,-$, and summation over repeated indices $(A$ as well as $a$ ) is understood.

Let us furthermore assume that the realization of the Poincare group that we discussed in the previous section is canonical relatively to $\sigma$. This implies that $\sigma$ is Poincaré invariant or, equivalently,

$$
\mathscr{L}\left(\mathbf{P}_{\bar{A}}\right) \sigma=0, \quad \mathscr{L}\left(\mathbf{J}_{\overline{A B}}\right) \sigma=0,
$$

where $\bar{A}, \bar{B}=1,2,-,+$ and $\mathscr{L}$ means "Lie derivative."

As a consequence of Eq. (3.2), there exist ten generating functions $P_{\bar{A}}(x, p), J_{\overline{A B}}(X, p)$ such that

$$
i\left(\mathbf{P}_{\bar{A}}\right) \sigma=-d P_{\bar{A}} \quad \text { and } \quad i\left(\mathbf{J}_{\overline{A B}}\right) \sigma=-d J_{\overline{A B}},
$$

or

$$
\mathbf{P}_{\bar{A}}=\left\{P_{\bar{A}},-\right\} \text { and } \mathbf{J}_{\overline{A B}}=\left\{J_{\overline{A B}},-\right\},
$$

where $i$ means "inner product" and $\{$,$\} is the Poisson$ bracket associated to $\sigma$.

Now, using Eq. (3.3) and the expressions (2.15) and (2.16) for the Poincaré generators, we arrive at

$$
\begin{aligned}
& \left\{x_{a}^{A}, P_{B}\right\}=\delta_{B}^{A}, \quad\left\{x_{a}^{A}, J_{12}\right\}=-x_{a}^{2} \delta_{1}^{A}+x_{a}^{i} \delta_{z}^{A}, \\
& \left\{x_{a}^{A}, J_{+}\right\}=x_{a}^{-} \delta_{-}^{A}, \quad\left\{x_{a}^{A}, J_{r-}\right\}=x_{a}^{r} \delta_{-}^{A}, \\
& \left\{x_{a}^{A}, J_{+r}\right\}=x_{a}^{-} \delta_{r}^{A}+x_{a}^{r} v_{a}^{A}, \quad\left\{x_{a}^{A}, P_{+}\right\}=-v_{a}^{A}, \\
& \quad r=1,2, \quad A, B=1,2,-.
\end{aligned}
$$

By applying the commutation relation of the Poincare algebra and using the Jacobi identity and Eqs. (3.4), we find, after some calculations,

$$
\begin{aligned}
\left\{J_{+r},\left\{x_{b}^{B}, x_{a}^{A}\right\}\right\}= & \delta_{r}^{i}\left\{x_{a}^{-}, x_{b}^{B}\right\}+v_{a}^{A}\left\{x_{a}^{r}, x_{b}^{B}\right\}+x_{a}^{r}\left\{v_{a}^{A}, x_{b}^{B}\right\} \\
& -v_{b}^{B}\left\{x_{b}^{r}, x_{a}^{A}\right\}-x_{b}^{r}\left\{v_{b}^{B}, x_{a}^{A}\right\} \\
\left\{x_{a}^{A}, v_{b}^{B}\right\}=\left\{x_{b}^{B}, v_{a}^{A}\right\} & +\left\{\left\{x_{b}^{B}, x_{a}^{A}\right\}, P_{+}\right\},
\end{aligned}
$$




$$
\begin{aligned}
&\left\{x_{a}^{A}, a_{b}^{B}\right\}=\left\{v_{b}^{B}, v_{a}^{A}\right\}+\left\{\left\{v_{b}^{B}, x_{a}^{A}\right\}, P_{+}\right\}, \\
&-\left\{\left\{x_{b}^{B}, v_{a}^{A}\right\}, J_{+}\right\}+\left\{x_{b}^{-}, v_{a}^{A}\right\} \delta_{r}^{B}+\left\{x_{a}^{r}, v_{a}^{A}\right\} \delta_{r}^{B} \\
&+\left\{x_{b}^{r}, v_{a}^{A}\right\} v_{b}^{B}+\left\{x_{b}^{B}, x_{a}^{A}\right\} x_{b}^{r} \\
&=\left\{v_{a}^{-}, x_{b}^{B}\right\} \delta_{r}^{A}+v_{a}^{r}\left\{v_{a}^{A}, x_{b}^{B}\right\}+v_{a}^{A}\left\{v_{a}^{r}, x_{b}^{B}\right\} \\
&-a_{a}^{A}\left\{x_{a}^{r}, x_{b}^{B}\right\}-x_{a}^{r}\left\{a_{a}^{A}, x_{b}^{B}\right\} \\
&-\{\left.\left\{v_{b}^{B}, v_{a}^{A}\right\}, J_{+r}\right\}+\left\{v_{b}^{-}, v_{a}^{A}\right\} \delta_{r}^{B}+\left\{v_{b}^{r}, v_{a}^{A}\right\} v_{b}^{B} \\
&+\left\{v_{b}^{B}, v_{a}^{A}\right\} v_{b}^{r}-\left\{x_{b}^{r}, v_{a}^{A}\right\} a_{b}^{B}-\left\{a_{b}^{B}, v_{a}^{A}\right\} x_{b}^{r} \\
&=\left\{v_{a}^{-}, v_{b}^{B}\right\} \delta_{r}^{A}+\left\{v_{a}^{r}, v_{b}^{B}\right\} v_{a}^{A} \\
&+\left\{v_{a}^{A}, v_{b}^{B}\right\} v_{a}^{r}-\left\{x_{a}^{r}, v_{b}^{B}\right\} a_{a}^{A}-\left\{a_{a}^{A}, v_{b}^{B}\right\} x_{a}^{r} .
\end{aligned}
$$

Since the coordinates $x_{a}^{A}$ are assumed to be canonical, we have that $\left\{x_{a}^{A}, x_{b}^{B}\right\}=0$, which, combined with (3.5) and (3.6), implies

$$
x_{a}^{r}\left\{v_{b}^{A}, x_{a}^{B}\right\}-x_{b}^{r}\left\{v_{b}^{A}, x_{A}^{B}\right\}=0,
$$

whence

$$
\left\{v_{b}^{A}, x_{a}^{B}\right\}=0, \quad \text { for } a \neq b .
$$

From (3.8), (3.10), and (3.11), we obtain

$$
\left\{a_{a}^{A}, x_{b}^{B}\right\}=0, \quad a \neq b,
$$

which, introduced into (3.7), yields

$$
\left\{v_{a}^{A}, v_{b}^{B}\right\}=0, a \neq b .
$$

Now, using the Jacobi identity with $v_{a}^{A}, v_{b}^{B}$, and $P_{+}$, we can write

$$
\left\{a_{a}^{A}, v_{b}^{B}\right\}=\left\{a_{b}^{B}, v_{a}^{A}\right\}+\left\{P_{+},\left\{v_{a}^{A}, v_{b}^{B}\right\}\right\},
$$

which, with the help of (3.13), leads to

$$
\left\{a_{a}^{A}, v_{b}^{B}\right\}=\left\{a_{b}^{B}, v_{a}^{A}\right\} .
$$

Upon substitution into (3.9), this finally yields

$$
\left\{a_{b}^{B}, v_{a}^{A}\right\}=0, a \neq b \text {. }
$$

Now taking into account the identity

$$
\{f, g\}=\sum_{i, j=1}^{k}\left\{\eta_{i}, \eta_{j}\right\} \frac{\partial f}{\partial \eta_{i}} \frac{\partial g}{\partial \eta_{j}},
$$

where $f$ and $g$ depend on the variables $\eta_{1} \cdots \eta_{k}$ only; we can write

$$
\begin{aligned}
& \left\{x_{a}^{A}, a_{b}^{B}\right\}=\left\{x_{a}^{A}, x_{c}^{D}\right\} \frac{\partial a_{b}^{B}}{\partial x_{c}^{D}}+\left\{x_{a}^{A}, v_{c}^{D}\right\} \frac{\partial a_{b}^{B}}{\partial v_{c}^{D}}, \\
& \left\{v_{a}^{A}, a_{b}^{B}\right\}=\left\{v_{a}^{A}, x_{c}^{D}\right\} \frac{\partial a_{b}^{B}}{\partial x_{c}^{D}}+\left\{v_{a}^{A}, v_{c}^{D}\right\} \frac{\partial a_{b}^{B}}{\partial v_{c}^{D}},
\end{aligned}
$$

whence, by using Eqs. (3.10)-(3.14) there follows that

$$
\frac{\partial a_{b}^{B}}{\partial v_{c}^{D}}=0, \quad \frac{\partial a_{b}^{B}}{\partial x_{c}^{D}}=0, \quad c \neq b .
$$

That is, the acceleration $a_{b}^{B}, B=1,2,-$, of each particle $b$ does not depend on the positions and velocities of the remaining ones, but only on its own position $x_{b}^{A}$ and velocity $v_{b}^{A}$. This conclusion would be enough to consider that the nointeraction result is proven, since the motion of each particle is not affected by the presence of the others. However, in the case we are considering (i.e., front form) a little bit deeper analysis reveals that the accelerations actually vanish.

Indeed, from $(2.15 \mathrm{~b})$ and $(3.17)$, we have

$$
\frac{\partial a_{a}^{B}}{\partial x_{a}^{A}}=0 \text {, }
$$

and using (2.16), (3.17), and (3.18), we arrive at

$$
a_{a}^{A}=0, \quad a=1, \ldots, N, \quad A=1,2,-,
$$

which completes the proof.

\section{POINT FORM OF PREDICTIVE RELATIVISTIC MECHANICS}

In the instant and front forms of dynamics, the construction of the configuration space was somehow linked to the choice of either the space hyperplanes $x^{4}=t$ or the null ones $x^{3}+x^{4}=\lambda$, respectively. In the point form, the hyperboloids $x^{\mu} x_{\mu}=-\lambda^{2}$ will be assigned a similar role.

Each point in the extended configuration space will be characterized by $3 N+1$ coordinates $\left(x_{a}^{i}, \lambda\right)$, $a=1, \ldots, N, i=1,2,3$, where $\lambda$ is taken as an evolution parameter and the $x_{a}^{i}$ are the spacelike coordinates of the event where the worldline of the ath particle intersects the hyperboloid

$$
x^{\mu} x_{\mu}=-\lambda^{2} .
$$

As in the earlier two cases, the equations of motion are second-order differential equations

$$
\frac{d^{2} x_{a}^{i}}{d \lambda^{2}}=a_{a}^{i}\left(x_{b}^{i}, v_{c}^{k}, \lambda\right), \frac{d x_{a}^{i}}{d \lambda}=v_{a}^{i},
$$

where $a, b, c=1, \ldots, N, i, j, k=1,2,3$.

Now, let $\varphi_{a}^{i}\left(x_{b}^{i}, v_{c}^{k}, \lambda_{0}, \lambda\right)$ be the general solution of (4.2) determined by the initial conditions

$$
\begin{aligned}
& \varphi_{a}^{i}\left(x_{b}^{j}, v_{c}^{k}, \lambda_{0} ; \lambda_{0}\right)=x_{a}^{i}, \\
& \dot{\varphi}_{a}^{i}\left(x_{b}^{i}, v_{c}^{k}, \lambda_{0} ; \lambda_{0}\right)=v_{a}^{i},
\end{aligned}
$$

where an overdot means a partial derivative with respect to the parameter $\lambda$. As in the former two cases, the worldline of the $a$ th particle is then defined by $\varphi_{a}^{\mu}\left(x_{b}^{j}, v_{c}^{k}, \lambda_{0} ; \lambda\right)$, where $\varphi_{a}^{0}\left(x_{b}^{j}, v_{c}^{k}, \lambda_{0} ; \lambda\right)=\left\{\lambda^{2}+\sum_{i=1}^{3} \varphi_{a}^{i}\left(x_{b}^{j}, v_{c}^{k}, \lambda_{0} ; \lambda\right)^{2}\right\}^{1 / 2}$.

And, as before, we shall require Poincaré invariance of worldlines that reads as

$$
\varphi_{a}^{\mu}\left(z_{0}^{\prime}, \lambda_{a}\left(z_{0}, \lambda\right)\right)=L^{\mu}{ }_{\nu}\left[\varphi_{a}^{\nu}\left(z_{0}, \lambda\right)-A^{\nu}\right],
$$

where $z_{0}$ and $z_{0}^{\prime}$ are abbreviations of the initial data, $\left(x_{b}^{j}, v_{c}^{k}\right.$, $\left.\lambda_{0}\right)$ and $\left(x_{b}^{\prime j}, v_{c}^{\prime k}, \lambda_{0}^{\prime}\right)$, respectively. These initial data correspond to two different inertial frames that are related to each other by the given Poincare transformation $\left(L^{\mu}{ }_{v}, A^{\rho}\right)$. The mapping $z_{0} \rightarrow z_{0}^{\prime}$ defines the induced transformation on the extended configuration space.

The value of the parameter $\lambda_{a}\left(z_{0}, \lambda\right)$ on the left-hand side of Eq. (4.5) can be easily derived. Indeed, taking (4.4) into account, we have

$\lambda_{a}^{2}\left(z_{0}, \lambda\right)=-\left[\varphi_{a}^{v}\left(z_{0}, \lambda\right)-A^{\mu}\right]\left[\varphi_{a v}\left(z_{0}, \lambda\right)-A_{v}\right]$. 
As is easily seen from this equation, and also from (4.4), the correspondence between $\lambda$ and the time coordinate $\varphi_{a}^{0}$ is not one-to-one. In order to avoid the nondifferentiability in the branch point $\lambda=0$, we shall take hereafter $\lambda_{0}$ and $\lambda$ positive. Moreover, the translation parameters $A^{\mu}$ will be assumed to be small enough for $\lambda_{a}^{2}\left(z_{0}, \lambda\right)$ on the left-hand side of $\mathrm{Eq}$. (4.6) to remain positive.

By differentiating (4.5) with respect to $\lambda$ we obtain the transformation formula for the velocities

$$
L^{\mu}{ }_{\nu} \dot{\varphi}_{a}^{\nu}\left(z_{0}, \lambda\right)=\dot{\varphi}_{a}^{\mu}\left(z_{0}^{\prime}, \lambda_{a}\left(z_{0}, \lambda\right)\right) \dot{\lambda}_{a}\left(z_{0}, \lambda\right),
$$

where

$\dot{\lambda}_{a}\left(z_{0}, \lambda\right)=\left[\lambda_{a}\left(z_{0}, \lambda\right)\right]^{-1}\left[\varphi_{a}^{\nu}\left(z_{0}, \lambda\right)-A^{\nu}\right] \dot{\varphi}_{a v}\left(z_{0}, \lambda\right)$.

And, differentiating again, we have the relationship among accelerations

$$
\begin{aligned}
L^{\mu}{ }_{\nu} \ddot{\varphi}_{a}^{v}\left(z_{0}, \lambda\right)= & \ddot{\varphi}_{a}^{\mu}\left(z_{0}^{\prime}, \lambda_{a}\left(z_{0}, \lambda\right)\right) \lambda_{a}^{2}\left(z_{0}, \lambda\right) \\
& +\dot{\varphi}_{a}^{\mu}\left(z_{0}^{\prime}, \lambda_{a}\left(z_{0}, \lambda\right)\right) \ddot{\lambda}_{a}\left(z_{0}, \lambda\right),
\end{aligned}
$$

where

$$
\begin{aligned}
\ddot{\lambda}_{a}= & -\frac{\dot{\lambda}_{a}^{2}}{\lambda_{a}}+\frac{1}{\lambda_{a}}\left\{\left(\varphi_{a}^{v}\left(z_{0}, \lambda\right)-A^{v}\right) \ddot{\varphi}_{a v}\right. \\
& \left.+\dot{\varphi}_{a}^{v}\left(z_{0}, \lambda\right) \dot{\varphi}_{a v}\left(z_{0}, \lambda\right)\right\} .
\end{aligned}
$$

Now using (4.3) and (4.4), taking (4.6)and (4.8) into account, and setting $\lambda=\lambda_{0}$, Eqs. (4.5) and (4.7) yield a set of $6 N$ implicit equations involving $x_{a}^{i}, v_{b}^{j}, x_{c}^{\prime k}, v_{d}^{\prime l}, \lambda_{0}, L^{\mu}{ }_{v}, A^{p}$. Similarly to the front form case, it will be generally impossible to derive explicit expressions

$$
x_{a}^{\prime i}=f_{a}^{i}\left(x, v, \lambda_{0} ; L, A\right), \quad v_{a}^{i i}=g_{a}^{i}\left(x, v, \lambda_{0} ; L, A\right)
$$

for the action induced by a finite Poincare transformation $\left(L^{\mu}{ }_{\nu}, A^{\rho}\right)$. However, by introducing the infinitesmial expressions

$L^{\mu}{ }_{v}=\delta^{\mu}{ }_{\nu}+\epsilon^{\alpha \beta}\left(\delta^{\mu}{ }_{\alpha} \eta_{v \beta}-\delta^{\mu}{ }_{\beta} \eta_{v \alpha}\right)+O\left(\epsilon^{2}\right), A^{\rho}=\epsilon^{\rho}$

into Eqs. (4.5) and (4.7), we can easily derive the following expressions from ten infinitesimal generators:

$$
\begin{aligned}
\mathbf{P}_{0}= & \sum_{a, i}\left\{\frac{\left(\lambda+\mathbf{x}_{a}^{2}\right)^{1 / 2}}{\lambda} v_{a}^{i} \frac{\partial}{\partial x_{a}^{i}}+\left[\frac { v _ { a } ^ { i } } { ( \lambda ^ { 2 } + \mathbf { x } ^ { 2 } ) ^ { 1 / 2 } } \left(1+\frac{\mathbf{x}_{a} \cdot \mathbf{v}_{a}}{\lambda}\right.\right.\right. \\
& \left.\left.\left.-\frac{\lambda^{2}+\mathbf{x}_{a}^{2}}{\lambda^{2}}\right)+\frac{\left(\lambda^{2}+\mathbf{x}_{a}^{2}\right)^{1 / 2}}{\lambda} a_{a}^{i}\right] \frac{\partial}{\partial v_{a}^{i}}\right\} \\
\mathbf{P}_{j}= & -\sum_{a, i}\left\{\left(\frac{x_{a}^{j} v_{a}^{i}}{\lambda}+\delta_{j}^{i}\right) \frac{\partial}{\partial x_{a}^{i}}\right. \\
& \left.+\frac{1}{\lambda}\left(x_{a}^{j} a_{a}^{i}+v_{a}^{i} v_{a}^{j}-\frac{v_{a}^{i} x_{a}^{j}}{\lambda}\right) \frac{\partial}{\partial v_{a}^{i}}\right\} \\
\mathbf{J}_{0 j}= & \sum_{a}\left\{\left(\lambda^{2}+\mathbf{x}^{2}\right)^{1 / 2} \frac{\partial}{\partial x_{a}^{j}}+\frac{\lambda+\mathbf{x}_{a} \cdot \mathbf{v}_{a}}{\left(\lambda^{2}+\mathbf{x}^{2}\right)^{1 / 2}} \frac{\partial}{\partial v_{a}^{j}}\right\} \\
\mathbf{J}_{i j}= & \sum_{a}\left\{x_{a}^{j} \frac{\partial}{\partial x_{a}^{i}}-x_{a}^{i} \frac{\partial}{\partial x_{a}^{j}}+v_{a}^{j} \frac{\partial}{\partial v_{a}^{i}}-v_{a}^{i} \frac{\partial}{\partial v_{a}^{j}}\right\}
\end{aligned}
$$

Finally, substituting the infinitesimal Poincaré transformation (4.11) into Eq. (4.9), and taking (4.10) and (4.12) into account, we arrive at

$$
\begin{aligned}
\mathbf{J}_{i j} a_{a}^{k}= & a_{a}^{j} \delta_{k}^{i}-a_{a}^{i} \delta_{k}^{j}, \\
\mathbf{J}_{0 l} a_{a}^{k}= & \frac{\delta_{l}^{k}}{\sqrt{\lambda^{2}+\mathbf{x}_{a}^{2}}}\left(1+\mathbf{x}_{a}^{2}+\mathbf{x}_{a} \mathbf{a}_{a}-\frac{\left(\lambda+\mathbf{x}_{a} \mathbf{x}_{a}\right)^{2}}{\lambda^{2}+\mathbf{x}_{a}^{2}}\right) \\
\mathbf{P}_{l} a_{a}^{k}= & \frac{2}{\lambda^{2}}\left(x_{a}^{l} a_{a}^{k}+v_{a}^{l} v_{a}^{k}-\frac{x_{a}^{l} v_{a}^{k}}{\lambda}\right) \\
& -\frac{1}{\lambda}\left(a_{a}^{l} v_{a}^{k}+2 v_{a}^{l} a_{a}^{k}+x_{a}^{l} \frac{\partial a_{a}^{k}}{\partial \lambda}\right) \\
\mathbf{P}_{0} a_{a}^{k}= & \frac{2}{\lambda\left(\lambda^{2}+\mathbf{x}_{a}^{2}\right)^{1 / 2}}\left(\lambda+\mathbf{x}_{a} \mathbf{v}_{a}-\frac{\left(\lambda^{2}+\mathbf{x}_{a}^{2}\right)}{\lambda}\right) a_{a}^{k} \\
& +\frac{1}{\lambda\left(\lambda^{2}+\mathbf{x}_{a}^{2}\right)^{1 / 2}}\left(\mathbf{v}_{a}^{2}+\mathbf{x}_{a} \mathbf{a}_{a}\right. \\
& -\frac{\mathbf{x}_{a} v_{a}}{\lambda}-\frac{\left(\lambda+\mathbf{x}_{a} \mathbf{v}_{a}\right)}{\lambda} \\
& \left.+\frac{2\left(\lambda^{2}+\mathbf{x}_{a}^{2}\right)}{\lambda^{2}}-\frac{\left(\lambda+\mathbf{x}_{a} \mathbf{v}_{a}\right)^{2}}{\left(\lambda^{2}+\mathbf{x}_{a}^{2}\right)}\right) v_{a}^{k} \\
& +\frac{\left(\lambda^{2}+\mathbf{x}_{a}^{2}\right)^{1 / 2}}{\lambda} \frac{\partial a_{a}^{k}}{\partial \lambda},
\end{aligned}
$$

where

$$
\mathbf{H}=\sum_{a, i}\left(v_{a}^{i} \frac{\partial}{\partial x_{a}^{i}}+a_{a}^{i} \frac{\partial}{\partial v_{a}^{i}}\right)+\frac{\partial}{\partial \lambda}
$$

is the infinitesimal generator of $\lambda$ evolution.

Equations (4.13) play a similar role as Currie-Hill conditions in the instant form of dynamics. It can be easily proven that they are equivalent to the following requirements.

(i) The generators $\mathbf{P}_{\mu}, \mathbf{J}_{\mu \nu}$ given by (4.12) generate a realization of the Poincare algebra on the space of initial data (i.e., their commutation relations are the suitable ones).

(ii) This realization is invariant under $\lambda$ evolution, that is,

$$
\left[\mathbf{H}, \mathbf{P}_{\mu}\right]=\left[\mathbf{H}, \mathbf{J}_{\mu v}\right]=0 .
$$

\section{THE NO-INTERACTION THEOREM IN THE POINT FORM OF DYNAMICS}

Let us now assume that by introducing some $3 \mathrm{~N}$ momenta $p_{a}^{i}\left(\mathrm{x}_{b}, \mathbf{v}_{c}, \lambda\right), a=1, \ldots, N, i=1,2,3$, the extended configuration space can be mapped onto the extended phase space, spanned by the $6 N+1$ independent variables $\left(x_{a}^{i}\right.$, $\left.p_{b}^{j}, \lambda\right)$. Let us further assume that the latter is endowed with the canonical structure defined by the elementary Poisson brackets

$$
\left\{x_{o}^{i}, x_{b}^{j}\right\}=\left\{p_{a}^{i}, p_{b}^{j}\right\}=0, \quad\left\{x_{a}^{i}, p_{b}^{j}\right\}=\delta_{a b} \delta^{i j}
$$

and the Poincare transformations as well as $\lambda$ evolution are both canonical relative to this structure.

The latter condition implies the existence of 11 generating functions $H, P_{\mu}, J_{\mu \nu}, \mu, v=1,2,3,4$, such that

$$
\mathbf{H}=\{H,\}, \quad \mathbf{P}_{\mu}=\left\{\boldsymbol{P}_{\mu},\right\}, \quad \mathbf{J}_{\mu \nu}=\left\{J_{\mu v},\right\},
$$


where $H, P_{\mu}$, and $J_{\mu \nu}$ are given by (4.14) and (4.12), respectively.

According to (4.12), (4.14), and (5.1), we have

$$
\begin{aligned}
& \left\{H, x_{a}^{i}\right\}=v_{a}^{i}, \\
& \left\{P_{0}, x_{a}^{i}\right\}=\left[\left(\lambda^{2}+\mathbf{x}_{a}^{2}\right)^{1 / 2} / \lambda\right] v_{a}^{i}, \\
& \left\{P_{j}, x_{a}^{i}\right\}=-x_{a}^{j} v_{a}^{i} / \lambda-\delta_{j}^{i}, \\
& \left\{J_{j k}, x_{a}^{i}\right\}=\delta_{j}^{i} x_{a}^{k}-\delta_{k}^{i} x_{a}^{j}, \\
& \left\{J_{0 j}, x_{a}^{i}\right\}=\left(\lambda^{2}+\mathbf{x}_{a}^{2}\right)^{1 / 2} \delta_{j}^{i},
\end{aligned}
$$

Now, writing down the Jocobi identity for $P_{j}, x_{a}^{i}, x_{b}^{k}$, taking (5.1) into account and using (5.2), we arrive at

$$
x_{a}^{j}\left\{x_{b}^{k}, v_{a}^{i}\right\}=x_{b}^{j}\left\{x_{a}^{i}, v_{b}^{k}\right\} .
$$

Applying the same treatment to the functions $P_{0}, x_{a}^{i}, x_{b}^{j}$, we have

$$
\left\{x_{b}^{j}, v_{a}^{i}\right\}=\left\{x_{a}^{i}, v_{b}^{j}\right\},
$$

which, once introduced in (5.4), implies

$$
\left\{x_{b}^{j}, v_{a}^{i}\right\}=0, a \neq b .
$$

Then, starting from the Jacobi indentity for $P_{j}, v_{a}^{i}, x_{b}^{j}$ and using (5.2), (5.3), and (5.6) we obtain

$$
x_{a}^{j}\left\{x_{b}^{k}, a_{a}^{i}\right\}=x_{b}^{j}\left\{v_{a}^{i}, v_{b}^{k}\right\},
$$

which, substituted in the Jacobi identity corresponding to $P_{0}, v_{a}^{i}, x_{b}^{j}$ leads us to

$$
\left\{x_{b}^{k}, a_{a}^{i}\right\}=0, \quad\left\{v_{b}^{k}, v_{a}^{i}\right\}=0, \quad a \neq b .
$$

Finally, using (5.8) in the Jacobi identity corresponding to $P_{j}, v_{a}^{i}, v_{b}^{k}$, we arrive at

$$
\left\{v_{b}^{k}, a_{a}^{i}\right\}=0, \quad a \neq b .
$$

Since the Poisson bracket has rank $6 N$ and the mapping $\left(x_{a}^{i}, v_{b}^{j}, \lambda\right) \rightarrow\left(x_{a}^{i}, P_{b}^{j}, \lambda\right)$ is assumed to have rank $6 N+1$, there follows from (5.8) and (5.9) that

$$
\frac{\partial a_{b}^{j}}{\partial x_{a}^{i}}=0, \quad \frac{\partial a_{b}^{j}}{\partial v_{a}^{i}}=0, \quad a \neq b,
$$

or, equivalently,

$$
a_{b}^{j}=a_{b}^{j}\left(x_{b}^{i}, v_{b}^{k}, \lambda\right),
$$

which means that the acceleration of the $b$ th particle does not depend on the state of motion of the remaining one. We can conclude that particles do not interact among themselves.

However, the no-interaction theorem we are intending to prove goes further still. Indeed, not only does it state that particles do not interact but also that their worldlines are straight.

The specific form of the acceleration $a_{b}^{i}$ can be determined by introducing $\left(5.10^{\prime}\right)$ into (4.13). This leads us to

$$
a_{b}^{i}=\frac{1}{\lambda}\left[1+\mathbf{v}_{b}^{2}-\frac{\left(\lambda+\mathbf{x}_{b} \cdot \mathbf{v}_{b}\right)^{2}}{\lambda^{2}+\mathbf{x}_{b}^{2}}\right] v_{b}^{i},
$$

which, since the acceleration is proportional to the velocity, implies that motions are rectilinear. A suitable reparametrization of trajectories will yield uniform motions, and therefore, the proof is complete.

In fact, the a priori knowledge that the above-mentioned suitable parameter will be the "physical time" $\varphi_{a}^{0}$, and its relationship (4.4) to the "unphysical" scalar parameter $\lambda$, will allow us to derive the general solution of Eq. (5.11). Indeed, we can easily prove that

$$
\mathbf{c}+\mathbf{b} T\left(\lambda, \lambda_{0}, \mathbf{c}, \mathbf{b}\right) \text {, }
$$

with

$$
\begin{aligned}
T= & \left(1-\mathbf{b}^{2}\right)^{-1}\left\{\mathbf{b} \cdot \mathbf{c}-b_{0}-\left(\left(\mathbf{b} \cdot \mathbf{c}-t_{0}\right)^{2}\right.\right. \\
& \left.\left.+\left(\lambda^{2}-\lambda_{0}^{2}\right)\left(1-\mathbf{b}^{2}\right)\right)^{1 / 2}\right\}
\end{aligned}
$$

and

$$
t_{0}=\left(\lambda_{0}^{2}-\mathbf{c}^{2}\right)^{1 / 2}
$$

is the general solution of Eq. (5.11); the parameters b.c being related to the initial data $\underset{0}{\mathbf{x}_{b}} \underset{0}{\mathbf{v}_{b}}$ according to

$$
c=\mathbf{x}_{0}, \quad b=\frac{t_{0}}{\lambda_{0}+\mathbf{x}_{0} \mathbf{v}_{0}} \cdot \mathbb{v}_{0} .
$$

\section{APPENDIX A}

In this Appendix we present the most useful expressions concerning the action of the Poincare group on the Minkowski space $M_{4}$, in terms of the set of coordinates and parameters which are most suitable for the null plane formalism (or front form). Throughout this paper, we take $c=1$ and $\eta_{\mu \nu}=(+++-), \mu, v=1,2,3,4$.

If $x_{a}^{\mu}, \mu=1,2,3,4$, are the Cartesian coordinates of an event in $M_{4}$, then the Poincare transformation $\left(L^{\mu}{ }_{v}, A^{\mu}\right)$ changes them into

$$
x^{\prime \mu}=L^{\mu}{ }_{\nu}\left(x^{\nu}-A^{\nu}\right)
$$

A proper orthochronous Poincaré transformation $\left(L^{\mu}{ }_{\nu}, A^{\nu}\right)$ is characterized by ten parameters $\left(\epsilon^{\lambda}, \epsilon^{\mu \nu}\right)$, $\lambda, \mu, v=1,2,3,4, \mu<v$. In the standard parametrization, and for infinitesimal values of these parameters, we have that

$$
\begin{aligned}
& L^{\alpha}{ }_{\beta}=\delta^{\alpha}{ }_{\beta}+\epsilon^{\mu \nu}\left(\delta^{\alpha}{ }_{\mu} \eta_{\nu \beta}-\delta^{\alpha}{ }_{\nu} \eta_{\mu \beta}\right)+O\left(\epsilon^{2}\right), \\
& A^{\alpha}=\epsilon^{\mu} \delta^{\alpha}{ }_{\mu} .
\end{aligned}
$$

Hence, in Cartesian coordinates, and in the standard parametrization, the infinitesimal generators are

$$
\mathbf{P}_{\mu}=-\frac{\partial}{\partial x^{\mu}}, \quad \mathbf{J}_{\mu \nu}=x_{v} \frac{\partial}{\partial x^{\mu}}-x_{\mu} \frac{\partial}{\partial x^{\nu}} .
$$

In the front form, it is more convenient to use the new adapted coordinates

$$
x^{4}=M_{\mu}^{\bar{A}} x^{\mu}, \quad \bar{A}=1,2,+,-,
$$

where

$x^{+}=x^{3}+x^{4}, \quad x^{-}=\frac{1}{2}\left(x^{3}+x^{4}\right), \quad x^{1}=x^{1}, \quad x^{2}=x^{2}$,

that is,

$$
M_{\mu}^{\bar{A}_{\mu}}=\left(\begin{array}{cccc}
1 & 0 & 0 & 0 \\
0 & 1 & 0 & 0 \\
0 & 0 & 1 & 1 \\
0 & 0 & 1 / 2 & -1 / 2
\end{array}\right)
$$


In these coordinates, the Minkowski metric is given by

$$
\begin{aligned}
\eta_{\overline{A B}}= & \left(M^{-1}\right)^{\mu}{ }_{\bar{A}}\left(M^{-1}\right)^{\nu}{ }_{\bar{B}} \eta_{\mu \nu} \\
& =\left(\begin{array}{lllll}
1 & 0 & 0 & 0 \\
0 & 1 & 0 & 0 \\
0 & 0 & 0 & 1 \\
0 & 0 & 1 & 0
\end{array}\right) .
\end{aligned}
$$

From this expression it is obvious that

$$
M_{\bar{A}^{\mu}} \equiv \eta_{\overline{A B}} M^{\bar{B}}{ }_{\nu} \eta^{\mu \nu}=\left(M^{-1}\right)^{\mu}{ }_{\bar{A}} .
$$

So, the lowering and raising of indices works as

$$
M^{1}=M_{1}, \quad M^{2}=M_{2}, \quad M^{+}=M_{-}, \quad M^{-}=M_{+} .
$$

Expressed in these new coordinates, the transformation (A1) reads

$x^{x^{\bar{A}}}=\mathscr{L}_{\bar{B}}^{\bar{A}}\left(x^{\bar{B}}-\mathscr{A}^{\bar{B}}\right), \quad \bar{A}, \bar{B}=1,2,+,-$,

where

$$
\mathscr{L}^{\bar{A}} \bar{B}_{\bar{B}} \equiv M^{\bar{A}_{\mu}} L^{\mu}{ }_{\nu}\left(M^{-1}\right)^{\nu}{ }_{\bar{B}}, \quad \mathscr{A}^{\bar{B}} \equiv M^{\bar{B}}{ }_{\nu} \bar{A}^{\nu} .
$$

According to these definitions, and performing the change of parameters,

$$
\epsilon^{\bar{A}}=M_{\mu}^{\bar{A}} \epsilon^{\mu}, \quad \epsilon^{\overline{A B}}=\epsilon^{\mu \nu} M_{\mu}^{\bar{A}} M^{\bar{B}}{ }_{\nu},
$$

the infinitesimal expressions (A2) transform into

$$
\begin{aligned}
& \mathscr{L}^{\bar{A}} \overline{\bar{B}}=\delta^{\bar{A}} \bar{B}_{\bar{B}}+\epsilon^{\overline{C D}}\left(\delta^{\bar{A}} \bar{C} \eta_{\overline{D B}}-\delta^{\bar{A}} \eta_{\overline{C B}}\right)+O\left(\epsilon^{2}\right), \\
& \mathscr{A}^{\bar{A}}=\epsilon^{\bar{C}} \delta_{\bar{C}}^{\bar{A}} .
\end{aligned}
$$

Hence, the corresponding generators are

$$
\mathbf{P}_{\bar{A}}=-\frac{\partial}{\partial x^{A}}, \quad \mathbf{J}_{\overline{A B}}=x_{\bar{B}} \frac{\partial}{\partial x^{\bar{A}}}-x_{\bar{A}} \frac{\partial}{\partial x^{\bar{B}}} .
$$

The relationship between the two sets of generators (A3) and (A13) can be easily derived from their definitions and Eq. (A11); this relationship being

$$
\mathbf{P}_{\bar{A}}=M_{\bar{A}}^{\mu} \mathbf{P}_{\mu}, \quad \mathbf{J}_{\overline{A B}}=M_{\bar{A}}^{\mu} M_{\bar{B}}^{v} \mathbf{J}_{\mu v} .
$$

Notice that the coordinates $x^{\bar{A}}, \bar{A}=1,2,+,-$, are specially suitable to work in the instant form approach, since the null hyperplane equation $x^{3}=x^{4}=0$ is written in the new coordinates $x^{+}=0$. Moreover, the generators $\mathbf{P}_{\bar{A}}$ and $\mathbf{J}_{\overline{A B}}$ split in a natural way into kinematic ones (those pre- serving the null hyperplane)

$$
\mathbf{P}_{-}, \mathbf{P}_{1}, \mathbf{P}_{2}, \mathbf{J}_{12}, \mathbf{J}_{1-}, \mathbf{J}_{2-}, \mathbf{J}_{+-}
$$

and dynamic ones

$$
\mathbf{P}_{+}, \mathbf{J}_{1+}, \mathbf{J}_{2+} \text {. }
$$

\section{APPENDIX B}

In the future sheet of the light cone in Minkowski space defined by

$$
x^{4}>0, \quad x^{\mu} x_{\mu}<0,
$$

we introduce the following coordinates:

$$
y^{i}=x^{i}, \quad i=1,2,3, \quad y^{4}=\left(-x^{\mu} x_{\mu}\right)^{1 / 2} .
$$

In terms of these coordinates, the generators of infinitesimal Poincaré transformations are

$$
\begin{aligned}
& \mathbf{P}_{\mu}=-\frac{\left(\left(y^{4}\right)^{2}+\sum_{i}\left(y^{i}\right)^{2}\right)^{1 / 2}}{y^{4}} \frac{\partial}{\partial y^{4}}, \\
& \mathbf{P}_{i}=\frac{y^{i}}{y^{4}} \frac{\partial}{\partial y^{4}}-\frac{\partial}{\partial y^{i}}, \\
& \mathbf{J}_{0 i}=\left(\left(y^{4}\right)^{2}+\sum_{j}\left(y^{j}\right)^{2}\right)^{1 / 2} \frac{\partial}{\partial y^{i}}, \\
& \mathbf{J}_{i j}=y^{j} \frac{\partial}{\partial y^{i}}-y^{i} \frac{\partial}{\partial y^{j}} .
\end{aligned}
$$

'P. A. M. Dirac, Rev. Mod. Phys. 21, 392 (1949)

${ }^{2}$ L. H. Thomas, Phys. Rev. 85, 868 (1952); B. Bakamjian and L. H. Thomas, Phys. Rev. 92, 1300 (1953); L. L. Foldy, Phys. Rev. 122, 275 (1961). ${ }^{3}$ D.G. Currie, T. F. Jordan, and E. C. G. Sudarshan, Rev. Mod. Phys. 35, 350 (1963); H. Leutwyler, Nuovo Cimento 37, 556 (1965).

${ }^{4}$ D. G. Currie, Phys. Rev. 142, 817 (1966); R. N. Hill, Math. Phys. 8, 201 (1967); E. H. Kerner, Math. Phys. 9, 222 (1968). All the above references can be found in The Theory of Action at a Distance in Relativistic Particle Dynamics, edited by E. H. Kerner (Gordon and Breach, New York, 1972). Also, P. Droz-Vincent, Phys. Scr. 2, 129 (1970); L. Bel, Ann. Inst. H. Poincaré XII, 307 (1970); XIV, 189 (1971).

${ }^{5}$ R. N. Hill, Math. Phys. 8, 1756 (1967).

${ }^{6}$ P. A. M. Dirac, Rev. Mod. Phys. 21, 392 (1949).

${ }^{7}$ H. Leutwyler and J. Stern, Ann. Phys. 112, 94 (1978).

${ }^{8}$ L. Bel, Ann. Inst. H. Poincaré XII, 307 (1970); XIV, 189 (1971). 